\title{
Ant Colony Optimization Based Type-2 Fuzzy Force-Position Control for Backhoe Excavator Robot
}

\author{
Mohammed Y. Hassan \\ Control and Systems Eng. Dep. \\ University of Technology, Baghdad, IRAQ \\ myhazawy@yahoo.com.
}

\author{
Athraa Faraj Sugban \\ Control and Systems Eng. Dep. \\ University of Technology, Baghdad, IRAQ \\ a_athraa2014@yahoo.com
}

\begin{abstract}
Received: 12-Feb.-2017
http://doi.org/10.29194/NJES21010001

Abstract

This paper proposes the design and simulation of Interval Type-2 Fuzzy Logic Control using MATLAB/Simulink to control the position of the bucket of the backhoe excavator robot during digging operations. In order to reach accurate position responses with minimum overshoot and minimum steady state error, Ant Colony Optimization (ACO) algorithm is used to tune the gains of the position and force parts for the forceposition controllers to obtain the best position responses. The joints are actuated by the electrohydraulic actuators. The force-position control incorporating two-Mamdani type-ProportionalDerivative-Interval Type-2 Fuzzy Logic Controllers for position control and 3Proportional-Derivative Controllers for force control. The nonlinearity and uncertainty in the model that inherit in the electro hydraulic actuator system are also studied. The nonlinearity includes oil leakage and frictions in the joints. The friction model is represented as a Modified LuGre friction model in actuators. The excavator robot joints are subjected to Coulomb, viscous and stribeck friction. The uncertainty is represented by the variation of bulk modulus. It can be shown from the results that the ACO obtain the best gains of the controllers which enhances the position responses within the range of $(19,23 \%)$ compared with the controllers tuned manually.
\end{abstract}

Keywords: Backhoe excavator robot, ForcePosition control, IT2FLC, Ant Colony Optimization.

\section{Introduction}

Excavator is an earth moving machine which has four parts: Swing, Boom, Arm, and Bucket. A robotic excavator has a movable arm. It is used in construction sites. The types of excavators are: backhoe, dragline, suction excavator, long arm, power shovels and others. Excavators are used in many operations: mining excavations, digging, dumping and trenching, construction, powering building equipment, and roads. These operations use expensive equipment and work in dangerous environments. Mining companies can deal with problems of lack of labor by using robotic excavator which improves productivity, efficiency and operator safety [1, 2].

The nonlinear model of a 4-DOF (3 active joints) excavator robot consists of three parts; kinematics, dynamics and hydraulics. The dynamic model has been presented in the autonomous digging mode; therefore, the swing motion is ignored. The backhoe excavator robot is actuated by electro-hydraulic servo system. Hydraulic actuator has high power capability, smooth response characteristics, highly nonlinear dynamics model and positioning capability. Friction in the excavator robot joint was not taken into consideration in most of the previous works.

In recent years, critical reviews on backhoe excavator robot have become an important topic. Many researchers have dealt with the modeling and control of robotic excavator. Koivo et al. in 1996 developed a complete dynamic model using the Newton-Euler approach for three links mechanism of robotic excavator [3]. Nguyen in 2000 developed a controller (a fuzzy sliding control) and a new observer is used [4]. Frankel in 2004, described a commercial backhoe excavator. It has been modified for haptic control research at Georgia Tech's Fluid Power and Motion Control Center. Electro-hydraulic valves and feedback sensors have been modified to the haptic backhoe and interfaced with a haptic joystick through a computerized control system [5]. Mitrev et al. in 2011 described work related to Computer-aided design/Computer-aided manufacturing investigation of the mechanical system of large mining excavator with Tri-power system. The investigation is performed in Autodesk Inventor environment [6]. Furthermore, Patel in 2015 developed the mathematical model for dynamic excavator robot [7].

In this work, Interval Type-2 Fuzzy Logic control is used to control the position of the bucket backhoe excavator during digging operations. Design of force-position controller to overcome the nonlinearity inherent in the 4-DOF (3 active joints) for joints is explained when the detailed modelling of the 4-DOF ( 3 active joint) backhoe excavator robot actuated by electrohydraulic servo actuator system has been explained in details in references $[1,2,4]$. The design of the force-position controllers for 
the joints of the backhoe excavator robot incorporates two Proportional-Derivativeinterval-Type-2-Fuzzy Logic Control (2-PDIT2FLC) for position control and three Proportional-Derivative (3-PD) controllers for force control. These controllers are designed to improve the desired position specification such as minimum error in position, minimum overshoot, and minimum oscillation. Ant Colony Optimization (ACO) is used to tune the gains of the position and force parts of the controllers to obtain the best ones. However, Figure (1) shows the coordinate system assignments for backhoe robotic excavator and Figure (2) shows the block diagram of the nonlinear backhoe excavator robot model:

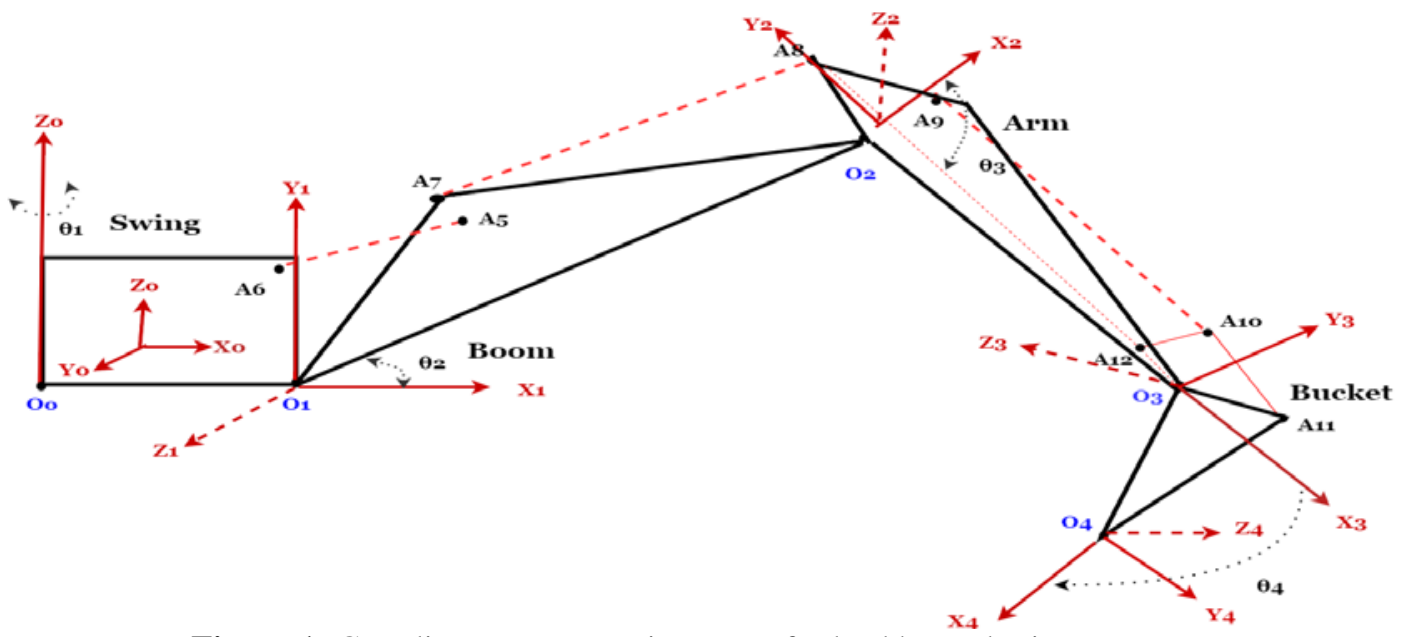

Figure 1: Coordinate system assignments for backhoe robotic excavator.

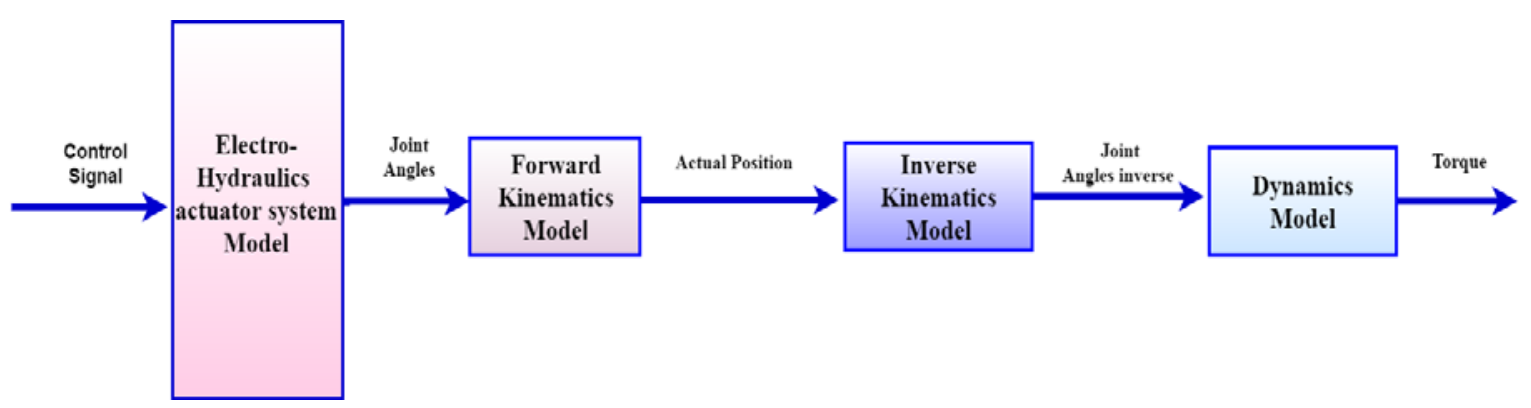

Figure 2: Block diagram the nonlinear backhoe excavator robot model.

Type-2 Fuzzy is a set of membership functions (MFs) that deal with the uncertainties in three dimensions. It was introduced by Mendel in 2001 [8].Type-2 Fuzzy sets membership functions are fuzzy and contain the Footprint of Uncertainty (FOU). It is capable of handling and modelling the numerical uncertainties, nonlinearities and linguistic associated with the inputs and output of the fuzzy logic control, by modelling them and reducing their effect. The structure of the T2FLC is shown in Figure (3):

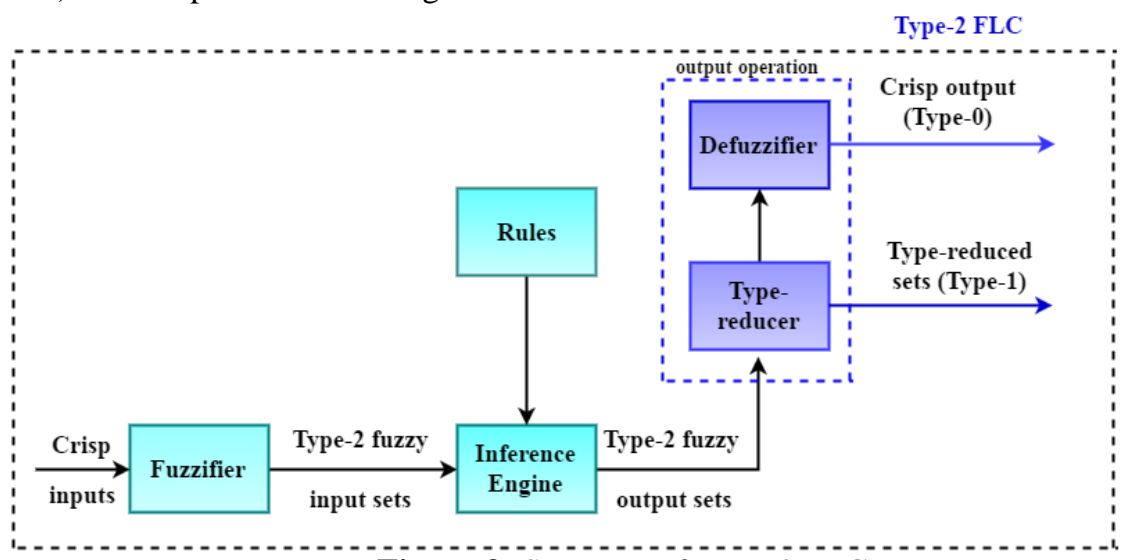

Figure 3: Structure of Type-2 FLC. 
There are two types of T2FLC: the Mamdani type and the Takagi-Sugeno-Kang (TSK) type. The differences between the two types are: the TSK membership fucntion is linear or constant and the Mamdani output membership is a shape. Furthermore, the Mamdani type needs type-reduction operations while the TSK doesn't need type-reduction operation. Methods used in type reduction operations include centroid, modified height, center of sum, center of sets, and height $[1,2]$.

\section{Design of Force-Position Control System}

The force-position controllers are designed for tracking the desired position trajectory for the backhoe excavator robot. The force-position control strategy take into consideration the relationship between the position of the manipulator and the contact forces imposed by the environment. Such an interaction wants the control of motion and interaction forces. This relationship can be modeled by generalized forceposition characterized by inertia, damping and stiffness properties, related to the manipulator [ 1 , $10,11]$. In this form, it is basically to use a PD position controller, with error and rate of error in position as inputs where the gains of the controller are adjusted in order to get different conspicuous force-positions. In this work, the gains of force-position controllers were tuned using proposed ACO algorithm [11, 12, 13]. Figure (4) shows the block diagram for the closed-loop backhoe excavator controlled system.

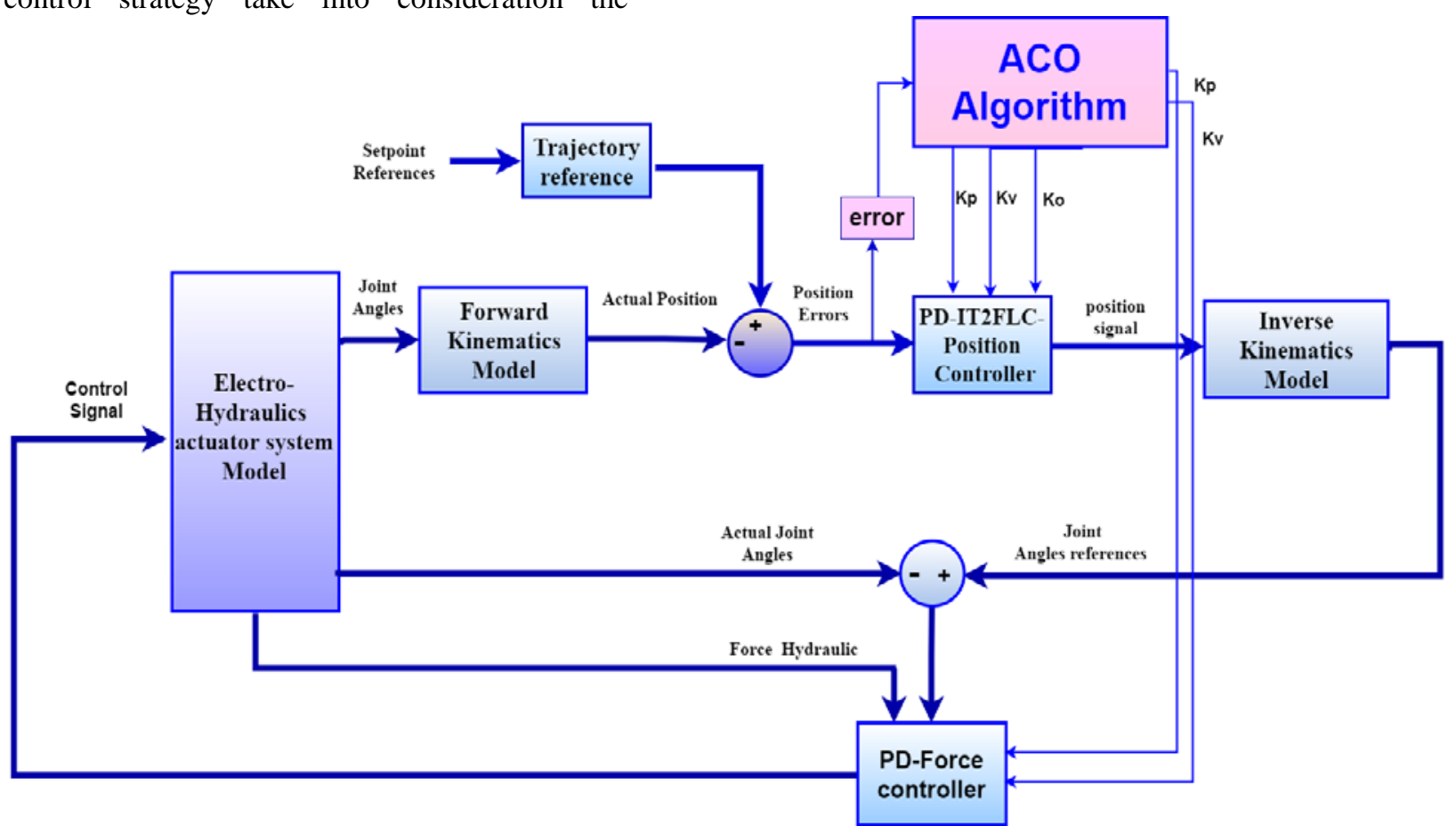

Figure 4: Closed loop force-position controlled system tuned by ACO.

The reference force for the boom, arm and bucket links are selected as [4]:

$F_{R}=M_{r}\left(\ddot{\theta}_{r}-\ddot{\theta}\right)+B_{r}\left(\dot{\theta}_{r}-\dot{\theta}\right)+K_{r}\left(\theta_{r}-\theta\right)+F_{f}(1)$ where $F_{f} \in R^{m} \times 1$ represents the resistance force from environment and $F_{R} \in R^{m} \times 1$ represents reference force, $F_{R}=\left[\begin{array}{lll}F_{r_{\text {Boom }}} & F_{r_{\text {Arm }}} & F_{r_{\text {Bucket }}}\end{array}\right]^{T}$ are the desired forces of the joints in (Newton), $F=\left[\begin{array}{lll}F_{\text {Boom }} & F_{\text {Arm }} & F_{\text {Bucket }}\end{array}\right]^{T}$ are the forces that represent the hydraulic forces of the joints in (Newton), $F_{\text {Boom }}$ represents the hydraulic force of the boom link, $F_{\text {Arm }}$ represents the hydraulic force of the arm link and $F_{\text {Bucket }}$ represents the hydraulic force of the bucket link. The parameters
$M_{r} \in R^{m \times m}, \quad B_{r} \in R^{m \times m}, \quad K_{r} \in R^{m \times m}$ are the positive constants. These parameters represent the inertia, damping and stiffness of each joint respectively. $\theta, \dot{\theta}$ and $\ddot{\theta}$ are the angular position, angular velocity and angular acceleration of the actual position of boom, arm and bucket respectively, $\theta_{r}, \dot{\theta}_{r}$ and $\ddot{\theta}_{r}$ represent the angular position, angular velocity and angular acceleration from the inverse kinematics model. They represent the desired joints of each link. However, Figure (5) shows the block diagram of the closed loop force controllers. 


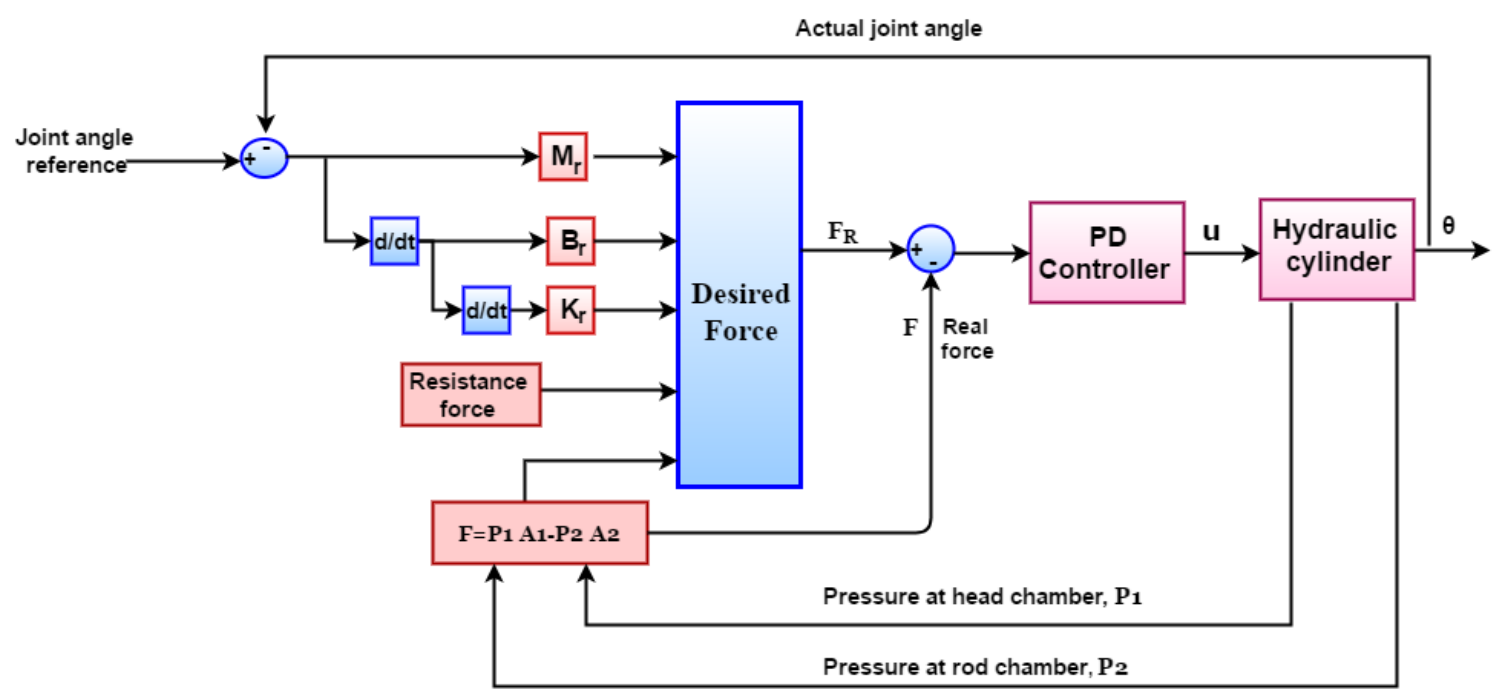

Figure 5: Closed loop force control system.

where (Actual joint angle) represents the joint angle for boom, arm and bucket links in (degree), the (Joint angle reference) represents the desired joint required for tracking the motion in (degree), and $u_{i}=\left[\begin{array}{lll}u_{1} & u_{2} & u_{3}\end{array}\right]^{T}$ are the control signals of the controllers in (Volt).

\section{Design of the Force-Position Controllers for Backhoe Excavator Robot}

Since the backhoe excavator robot and the hydraulic system are highly nonlinear, the forceposition controller has the ability to deal with the nonlinearity and uncertainty of the backhoe excavator robot and to track the desired position trajectory with minimum error in position response for force interaction.

The PD-Like Interval Type-2 Fuzzy Logic Controller is designed as a position controller. The inputs to IT2FLC are the error signal $e(t)$ and rate of error signal $\dot{e}(t)$. The position controller equation for joint $(i)$ is $[1,2]$ :

$$
u_{i}(t)=K_{P i} e(t)+K_{V i} \dot{e}(t)
$$

where the inputs of the IT2FLC and output scaling factors are defined as: Proportional gain.
$\left(K_{P i}\right)$, derivative gain $\left(K_{V i}\right)$, and output gain $\left(K_{O i}\right)$. The IT2FLC is of Mamdani type. The inputs and the output membership functions are selected as seven Gaussian shaped, as shown in Figure (6). The defuzzification technique is selected to be centroid method. Table (1) illustrates the rules of PD-like IT2FLC position controller. The choice of these rules is done using trial and error to reach the best response of the position.

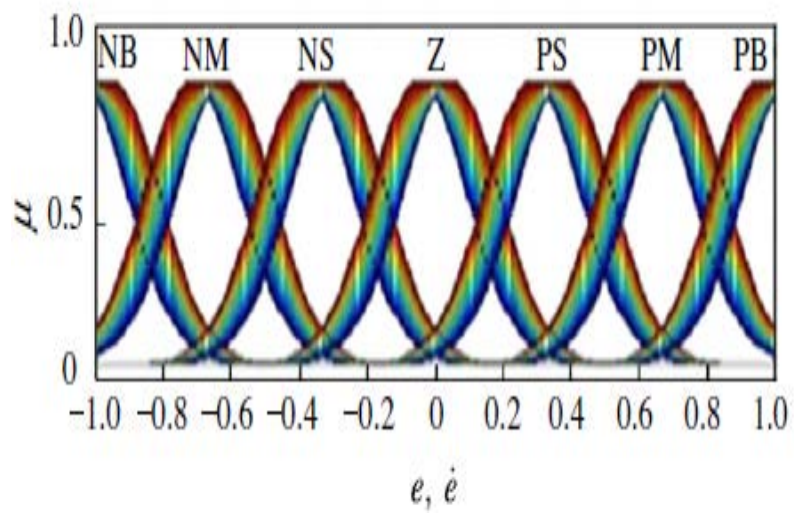

Figure 6: I/O membershi(2) functions of IT2FLC.

Table 1: Rules of PD-like position IT2FLC [2].

\begin{tabular}{|c|c|c|c|c|c|c|c|}
\hline $\boldsymbol{e} / \dot{\boldsymbol{e}}$ & NB & NM & NS & Z & PS & PM & PB \\
\hline NB & NB & NB & NB & NB & NM & NS & Z \\
\hline NM & NB & NB & NB & NM & NS & Z & PS \\
\hline NS & NB & NB & NM & NS & Z & PS & PM \\
\hline Z & NB & NM & NS & Z & PS & PM & PB \\
\hline PS & NM & NS & Z & PS & PM & PB & PB \\
\hline PM & NS & Z & PS & PM & PB & PB & PB \\
\hline PB & Z & PS & PM & PB & PB & PB & PB \\
\hline
\end{tabular}

The linguistic variables membership functions of the IT2FLC are: NB (Negative Big), NM (Negative Medium), NS (Negative Small), Z (Zero), PS (Positive Small), PM (Positive
Medium) and PB (Positive Big). The universe of discourse for the inputs and output are within ( -1 , 1). The parameters of the force controller incorporating in the force-position control are 
explained in equation (1). These values represent a second order reference model with a selected damping ratio $(\zeta)$ and un-damped natural frequency $\left(w_{n}\right)$ [10,11]. These parameters are selected to get the best desired forces, which are listed in Table (2). The Simulink of the position controlled system is shown in Figure (7) and the Simulink of force controlled system is shown in
Figure (8). However, The Simulink of the complete force-position controlled system for 3 DOF's backhoe excavator robot is shown in Figure (9). It consists of sub blocks of the kinematics model, dynamic model, hydraulic model, the position controller, the force controller, and the reference trajectory.

Table 2: Parameters of the force controller.

\begin{tabular}{|c|c|c|c|c|c|}
\hline Link of Excavator & $\boldsymbol{M}_{\boldsymbol{r}}(\boldsymbol{k g})$ & $\boldsymbol{B}_{\boldsymbol{r}}\left(\frac{\boldsymbol{N} . \boldsymbol{S}}{\boldsymbol{m}}\right)$ & $\boldsymbol{K}_{\boldsymbol{r}}\left(\frac{\boldsymbol{N}}{\boldsymbol{m}}\right)$ & $\zeta$ & $\boldsymbol{w}_{\boldsymbol{n}}\left(\frac{\boldsymbol{r a d}}{\boldsymbol{s e c}}\right)$ \\
\hline Bucket & 5 & 170 & 3040.34 & 0.69 & 24.66 \\
\hline Arm & 1 & 50 & 200 & 1.767 & 14.142 \\
\hline Boom & 5 & 200 & 3000 & 0.816 & 24.495 \\
\hline
\end{tabular}

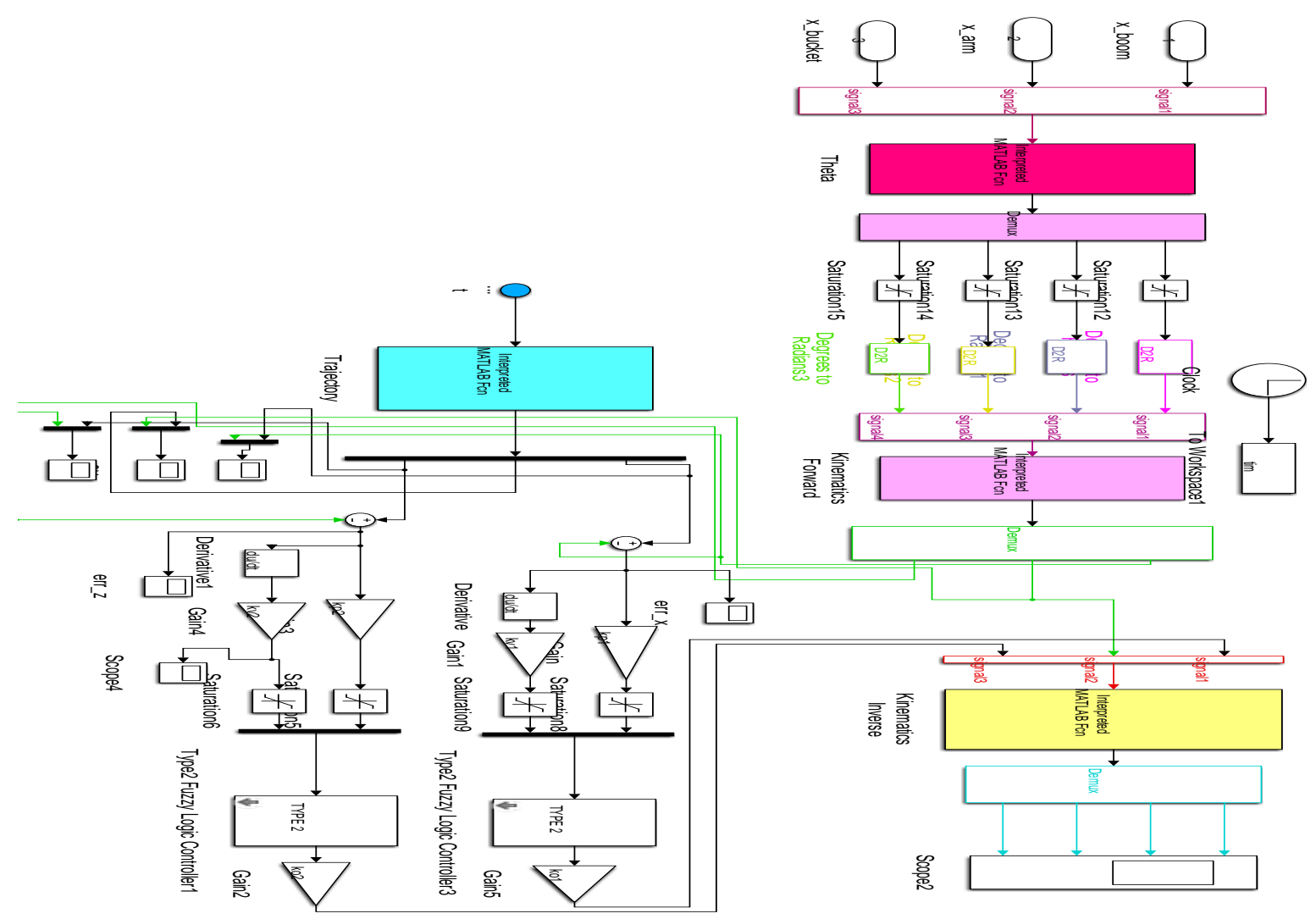

Figure 7: Simulink of the position controlled system. 

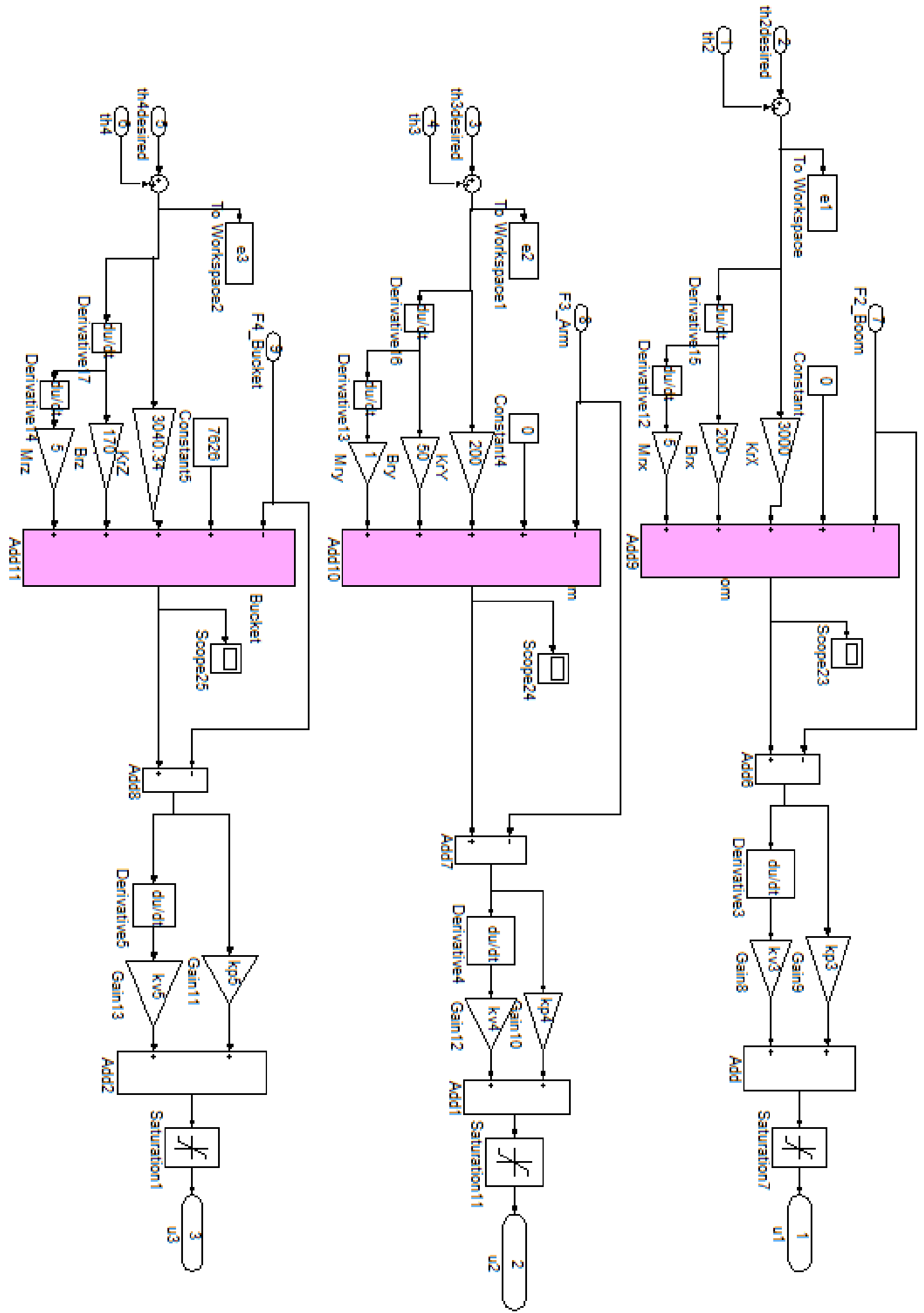

Figure 8: Simulink of force controlled system. 


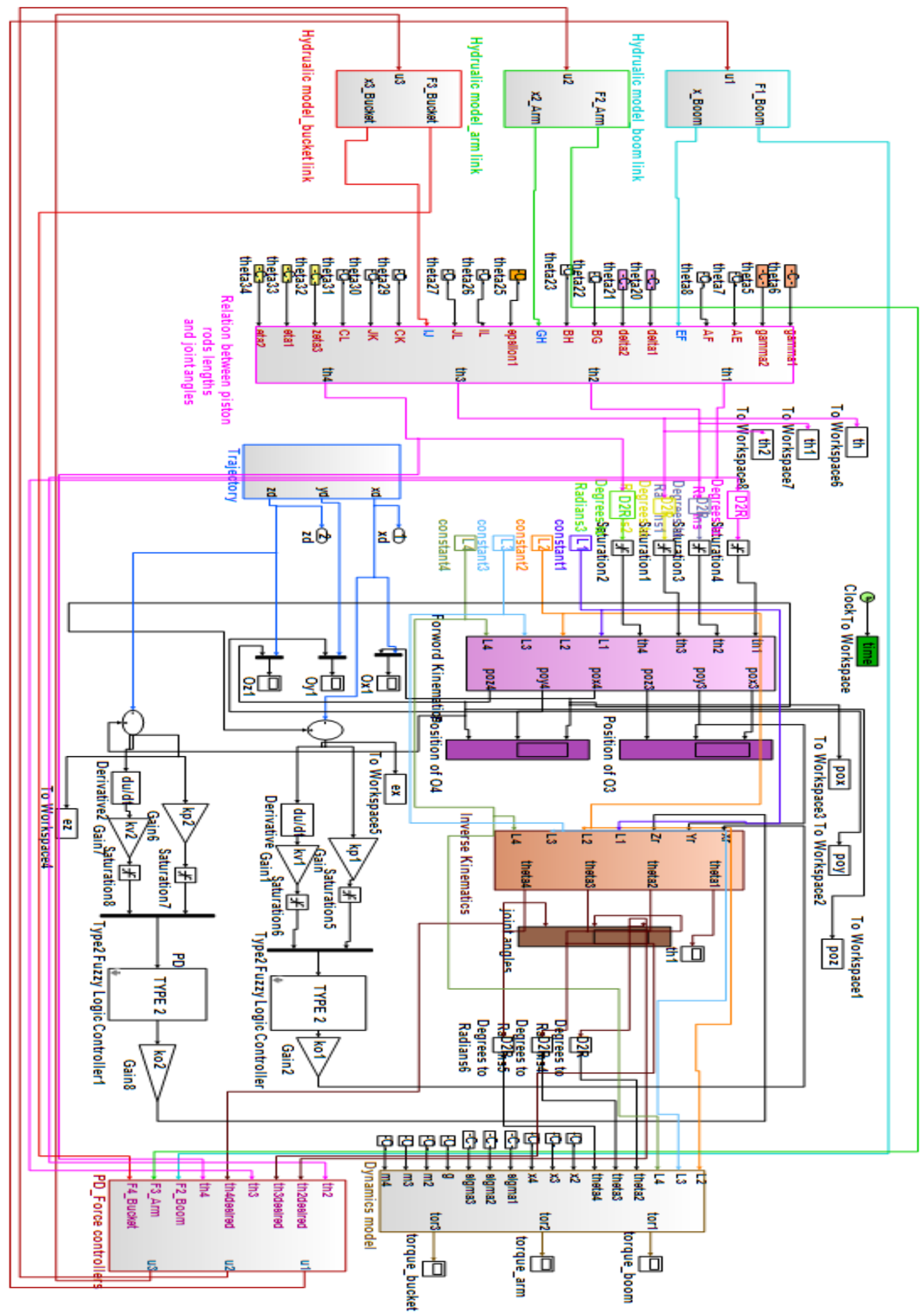

Figure 9: Simulink of the force-position controlled system of the backhoe excavator robot model. 


\section{Simulation Results}

In this work, trigonometric trajectories are applied. The trajectory is the path followed by the end-effector manipulator of the excavator robot and applying the time-domain along the path followed. The trajectory is transformed into Cartesian coordinates system using the forward kinematics. In order to obtain the desired position trajectory $X_{d}=\left[\begin{array}{lll}x_{d} & y_{d} & z_{d}\end{array}\right]^{T}$, the mathematical expression for trigonometric trajectory is used. The planed trigonometric trajectory has been explained in [7]. The trajectory is the time of planning the robot to arrive at the final position (end-effector) in total time of $\mathrm{T}_{\mathrm{t}}=10 \mathrm{sec}$.

By applying the reference position trajectory, the gains of the PD- IT2FLC of the position control and the gains of the PD-control of the force controller for each joint are manually tuned using several trials and error and the best values are listed in Table (3). The axes position responses are shown in Figures (10) and (11) respectively. The criterion to measure the best position response is using the Mean Square Error (MSE):

$$
M S E=\frac{1}{N} \sum_{i=1}^{N}\left(e_{x}^{2}(i)+e_{y}^{2}(i)+e_{z}^{2}(i)\right)
$$

where $e_{x}^{2}(i)$ is the error trajectory position in $\mathrm{x}$ axis, $e_{y}^{2}(i)$ is the error trajectory position in $y$ axis, $e_{z}^{2}(i)$ is the error trajectory position in $\mathrm{z}$ axis, and $N$ is the number of samples.

Table 3: PD-like force-position IT2FLC gains for the trigonometric trajectory.

\begin{tabular}{|c|c|}
\hline Gain & Value \\
\hline$K_{P 1}$ & 10 \\
\hline$K_{V 1}$ & 30 \\
\hline$K_{01}$ & 2 \\
\hline$K_{P 2}$ & 10 \\
\hline$K_{V 2}$ & 0.08 \\
\hline$K_{O 2}$ & 2 \\
\hline$K_{P 3}$ & 3 \\
\hline$K_{V 3}$ & 0.006 \\
\hline$K_{P 4}$ & 2 \\
\hline$K_{V 4}$ & 0.08 \\
\hline$K_{P 5}$ & 2 \\
\hline$K_{V 5}$ & 0.007 \\
\hline
\end{tabular}

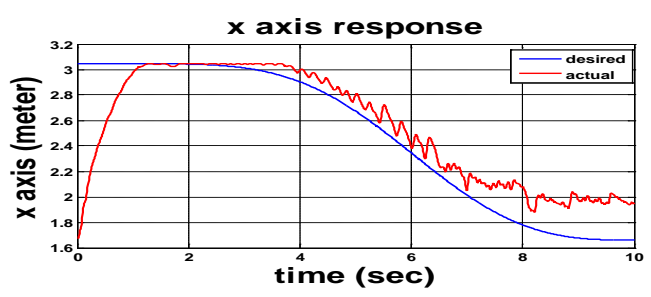

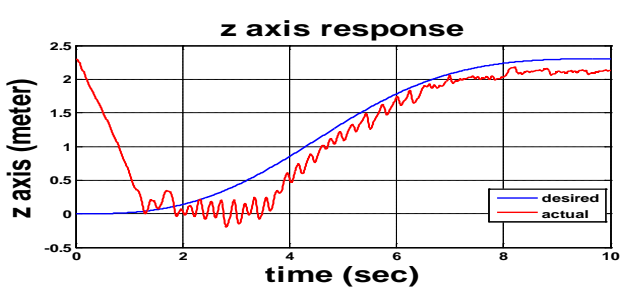

Figure 10: Position trajectory response for the $x$ and $\mathrm{z}$ axes.

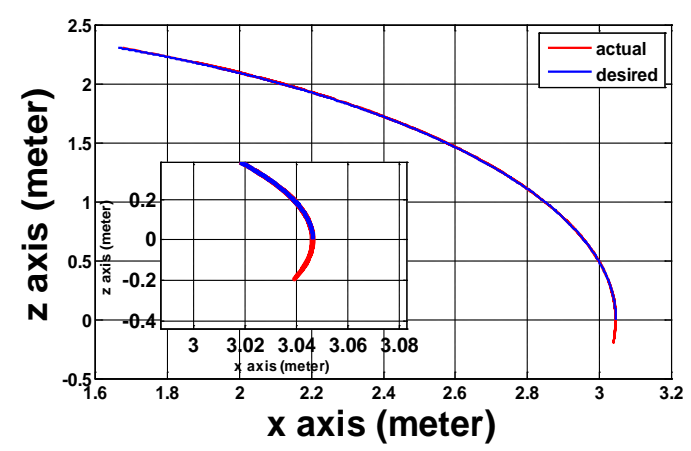

Figure 11: Position trajectory response.

The performance index (MSE) for position trajectory without changing bulk modulus and leakage is calculated and equals to 0.3817 . The effect of the nonlinearity and uncertainty appear clearly in the result that causes the errors between the reference trajectory and the actual position of each axis. It is clear from Figure (11) that the actual position trajectory does not coincide with the reference one.

The effects of changing bulk modulus and leakage are both studied. By increasing the leakage $15 \%$ and bulk modulus $40 \%$ from nominal value, the same gains of the controllers illustrated in Table (3) are used, the axes position responses are shown in Figure (12) and Figure (13). The Simulation results indicate the ability of the controller to control the system although changing the system parameters.
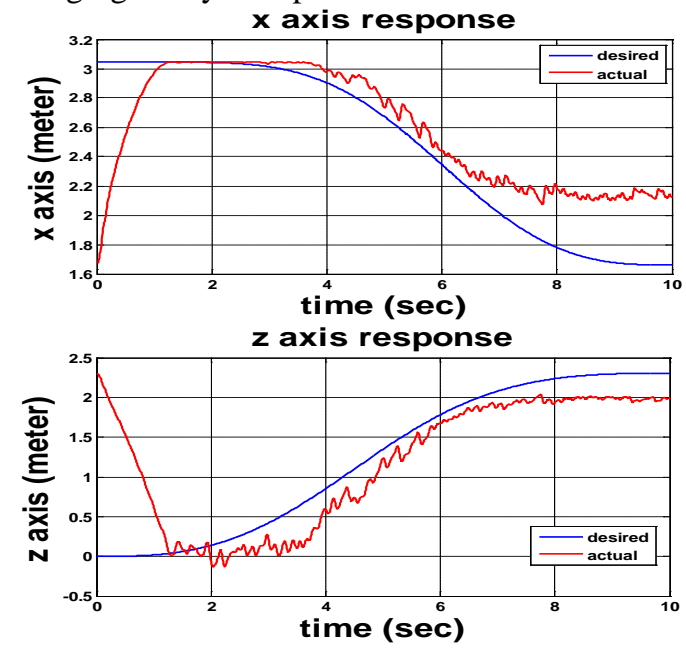

Figure 12: Position trajectory response for the $x$ and $\mathrm{z}$ axes with the effect of increasing bulk modulus and leakage 


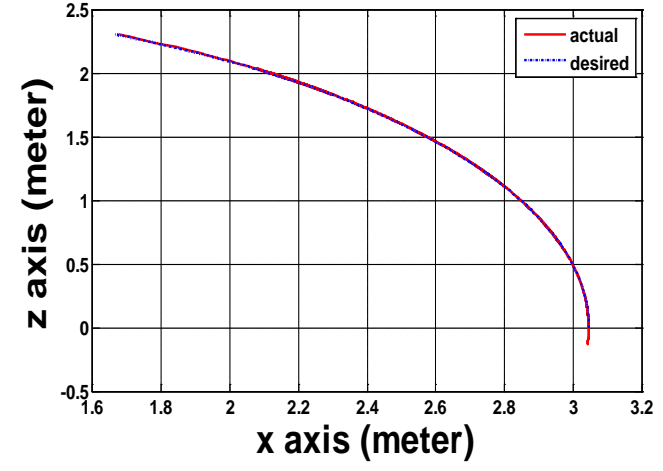

Figure 13: Position trajectory response with the effect of increasing both bulk modulus and leakage.

The performing index (MSE) for the position trajectory with the effects of bulk modulus and leakage change is calculated and equals to 0.4224 , the MSE is increasing because the effect of increasing both bulk modulus and leakage. When the value of MSE increases, this means increasing the error between the reference trajectory and the actual position, specially, in the end of trajectory. The match of the actual axis with the reference axis is not complete because of the effect of nonlinearity and uncertainty.

It is difficult to enhance position tracking performance using the controller gains tuned manually. The process of tuning the gains of the controllers consumes time and efforts. Also, the calculated MSE gives the indication that there is no match between the reference trajectory and the actual position inherent in the model.

Ant Colony Optimization (ACO) is used as an optimization algorithm to tune the gains of PDlike IT2FLC position and PD-like force controllers in order to reach the best position performance. This minimization gives closer matching between the desired and the actual trajectory.

To obtain more accurate position responses, the values of the gains $\left(K_{P i}, K_{V i}\right.$ and $K_{O i}$ ) for each position and force controllers are tuned by ACO to achieve the desired specifications of minimum error in position response and minimum overshoot. ACO uses a pheromone matrix $\tau=\left\{\tau_{i j}\right\}$ for the construction of potential good solutions. The initial values of $\tau$ are set as: $\tau_{i j}=\tau_{0} \forall(i, j)$, where $\tau_{0}>0$.

The probability $P_{i j}^{A}(t)$ of choosing a node $j$ at node $i$ is defined as $[12,13]$ :

$P_{i j}^{A}(t)=\frac{\left[\tau_{i j}\right]^{\alpha}\left[\eta_{i j}\right]^{\beta h}}{\sum_{l \in T^{A}}\left[\tau_{i j}\right]^{\alpha}\left[\eta_{i j}\right]^{\beta h}}, \quad$ if $j \in T^{A}$

The quantity of pheromone $\Delta \tau_{i j}^{A}$ on each path may be defined as:

$\Delta \tau_{i j}^{A}=\left\{\begin{array}{cc}\frac{L^{\min }}{L^{A}} & \text { if } i, j \in T^{A} \\ 0 & \text { else }\end{array}\right.$ where $L^{A}$ is the value of the objective function found by the ant $A$ and $L^{m i n}$ is the best solution carried out by the set of the ants until the current iteration.

The pheromone evaporation is a way to avoid unlimited increase of pheromone trails. Also, it allows the forgetfulness of the bad choices [12, 13]:

$\tau_{i j}(t)=\rho_{0} \tau_{i j}(t-1)+\sum_{A=1}^{N A} \Delta \tau_{i j}^{A}(t)$ where $\Delta \tau_{i j}^{A}$ is the quantity of pheromone on each path, NA is the number of ants and $\rho_{0}$ is the evaporation rate $0<\rho_{0} \leq 1$.

The best parameters got to be used by ACO method to reach minimum performance index, as illustrated in Table (4). These parameters have been used for carrying out the force-position controllers design using Ant Colony Optimization (ACO) for the position reference trajectory that was applied previously by using the Mean Square Error, equation (3):

Table 4: Best ACO parameters.

\begin{tabular}{|c|c|}
\hline Parameters & Trajectory \\
\hline No. of ants & 35 \\
\hline No. of Nodes & 10000 \\
\hline$\alpha$ & 0.8 \\
\hline$\beta h$ & 0.2 \\
\hline$\rho_{0}$ & 0.7 \\
\hline $\begin{array}{c}\text { No. of } \\
\text { iteration }\end{array}$ & 40 \\
\hline
\end{tabular}

where $K_{i}$ is the iteration number, $\alpha$ and $\beta h$ are constants that determine the relative influence of the pheromone values and the heuristic values on the decision of the ant. Many tests were done until reaching the best enhancement at minimum performance index and the largest enhancement at minimum fitness function. However, the gains of the force-position controllers obtained by the ACO are illustrated in Table (5). The performance index (MSE) for the trajectory is calculated and equals to 0.3106 .

Table 5: PD-like force-position IT2FLC gains for the trigonometric trajectory (ACO tuning).

\begin{tabular}{|c|c|}
\hline Gain & Value \\
\hline $\boldsymbol{K}_{\boldsymbol{P 1}}$ & 5.627 \\
\hline $\boldsymbol{K}_{\boldsymbol{V} \mathbf{1}}$ & 0.044 \\
\hline $\boldsymbol{K}_{\boldsymbol{O} \mathbf{1}}$ & 0.426 \\
\hline $\boldsymbol{K}_{\boldsymbol{P 2}}$ & 15.032 \\
\hline $\boldsymbol{K}_{\boldsymbol{V} \mathbf{2}}$ & 0.191 \\
\hline $\boldsymbol{K}_{\boldsymbol{O} \mathbf{2}}$ & 5.33 \\
\hline $\boldsymbol{K}_{\boldsymbol{P 3}}$ & 26.284 \\
\hline $\boldsymbol{K}_{\boldsymbol{V} \mathbf{3}}$ & 0.195 \\
\hline $\boldsymbol{K}_{\boldsymbol{P 4}}$ & 7.464 \\
\hline $\boldsymbol{K}_{\boldsymbol{V} \mathbf{4}}$ & 28.532 \\
\hline $\boldsymbol{K}_{\boldsymbol{P 5}}$ & 15.01 \\
\hline $\boldsymbol{K}_{\boldsymbol{V} \mathbf{5}}$ & 0.489 \\
\hline
\end{tabular}


The response of the position tracking responses in the $\mathrm{x}$ and $\mathrm{z}$ Cartesian space are shown in Figures (14) and (15) respectively. Figure (16) shows the performance index (fitness) obtained by ACO for the position trajectory.
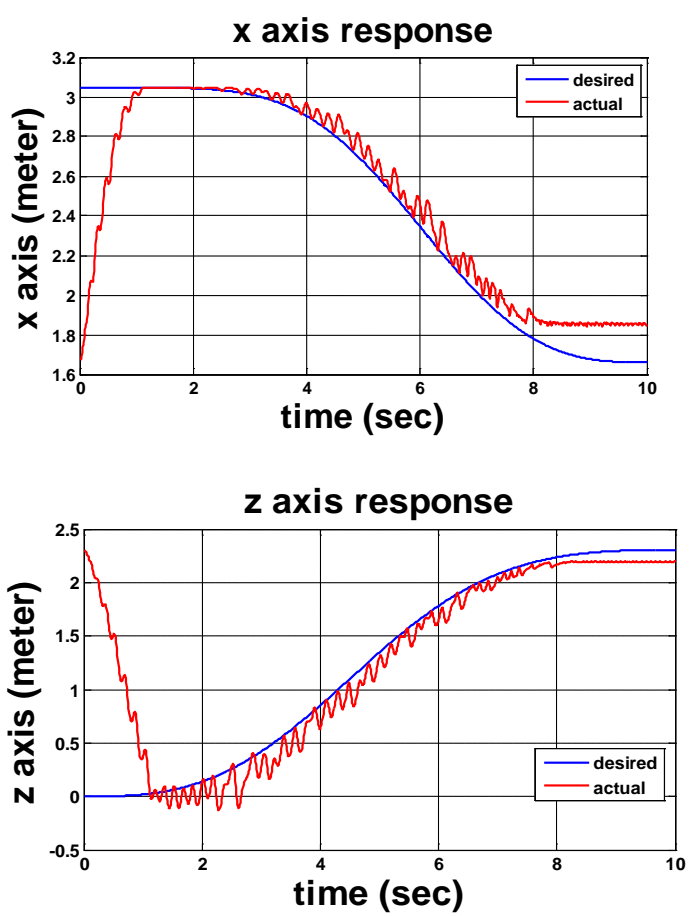

Figure 14: Position trajectory response for the $\mathrm{x}$ and $\mathrm{z}$ axes (ACO tuning).

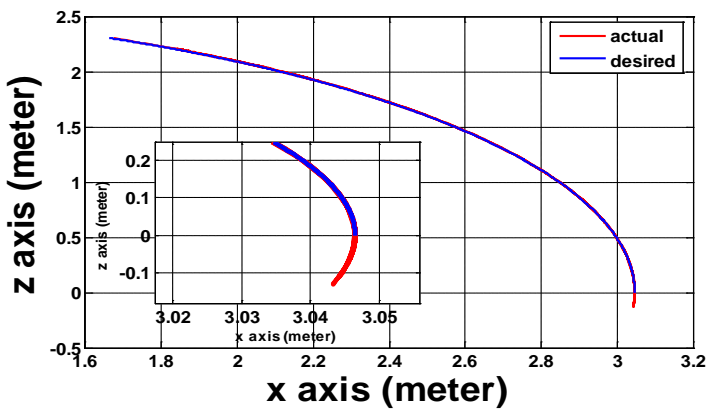

Figure 15: Position trajectory response (ACO tuning).

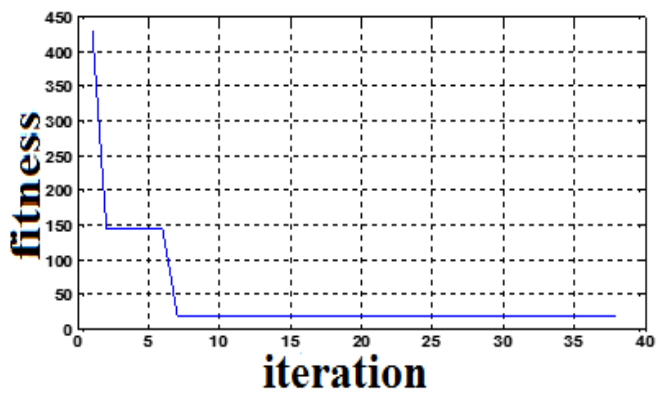

Figure 16: Performance index (fitness) obtained by ACO for the position trajectory.

The effects of bulk modulus and leakage change are also studied. By increasing the leakage
$15 \%$ and bulk modulus $40 \%$ from nominal values, using the gains of the controllers obtained by ACO tuning listed in Table (5), the axes position response with changing both the bulk modulus and leakage are shown in Figure (17) and Figure (18). There is a significant reduction in the error of position and the simulation results appear the ability of the ACO algorithm to control the system although changing the system parameters. The performing index (MSE) for the position trajectory with the effects of bulk modulus and leakage change is calculated and equals to 0.3433 .
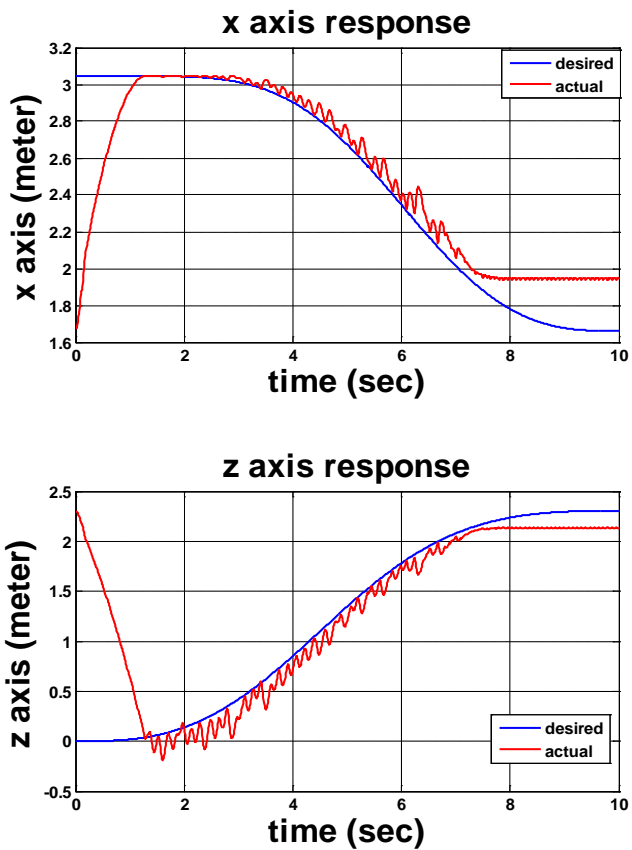

Figure 17: Position trajectory response for the $x$ and $\mathrm{z}$ axes with the effect of increasing both bulk modulus and leakage (ACO tuning).

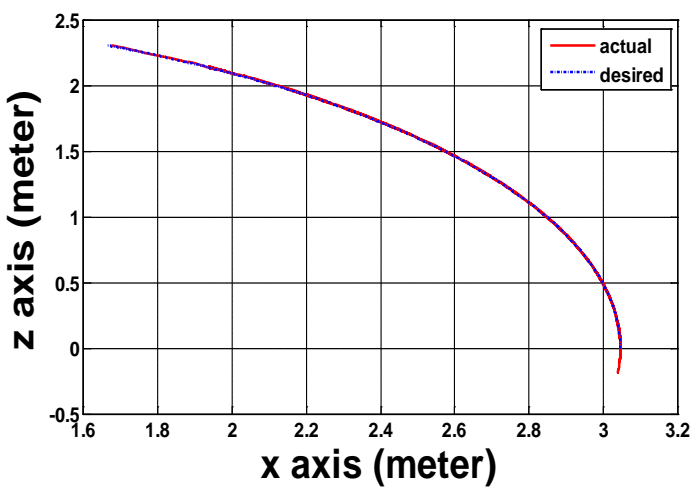

Figure 18: Position trajectory response with the effect of increasing both bulk modulus and leakage (ACO tuning).

\section{Conclusions}

Force-Position controllers were designed to control the proposed backhoe excavator robot in digging operations. The proposed controller using manual tuning could not accurately 
compensate the nonlinearity and uncertainty inherent in the model. Ant Colony Optimization (ACO) algorithm was used to tune the gains of the force and position controls of force-position controllers to satisfy the desired specification with minimum overshoot, minimum error in position response.

Results of applying trajectory under different conditions show an average enhancement in the position trajectory of $(19,23$ $\%)$ as compared with conventional controllers when the errors in position were determined using Mean Square Error (MSE) criteria. This work proves that the Interval Type-2 Fuzzy Intelligent control is able to minimize errors in position with and without the effect of nonlinearity and uncertainty in model by obtaining the best gains of the controllers.

\section{References}

[1] M. Y. Hassan and A. F. Sugban, "Nonlinear Modeling and IT2 Fuzzy Control Design of 4DOF Robotic Backhoe Excavator", The First International Conference for Engineering Researches (ICER), 2017.

[2] M. Y. Hassan and G. Kothapalli, "Interval Type-2 Fuzzy Position Control of Electrohydraulic Actuated Robotic Excavator", International Journal of Mining Science and Technology, Vol. 22, pp. 437-445, 2012.

[3] A. J. Koivo, M. Thoma , E. Kocao and J. Andrade-Cetto, "Modelling and control of excavator dynamic during digging operator", International Journal of Aerospace Engineering, Vol. 9, No. 1, January-1996.

[4] Q. H. Nguyen, "Robust Low Level Control of Robotic Excavator", Australia: University of Sydney, 2000.
[5] J. G. Frankel, "Development of a Haptic Backhoe Testbed", M. Sc. Thesis, Georgia Institute of Technology School of Mechanical Engineering, May, 2004.

[6] R. Mitrev, R. Gruychev, P. Pobegailo, "CAD/CAE Investigation of a Large hydraulic mining excavator", Machine Design, Vol. 3, No. 1, pp. 17-22, 2011.

[7] M. A. Patel, D. A. Patel, B. P. Patel, V. B. Patel, P. H. Shah," Trajectory Planning for Backhoe Excavator ", U V Patel College of Engineering, may-2015.

[8] J. M. Mendel, "Uncertain rule-based Fuzzy Logic Systems: Introduction and New Directions”, NJ: Prentice Hall PTR, 2001.

[9] M. B. Ozek and Z. H. Akpolat, "A Software Tool: Type-2 Fuzzy Logic Toolbox", Comput Appl Eng Educ, Vol. 16, No. 2, pp. 137-46, 2008.

[10] M. Y. Hassan and Z. A. Karam, "Modeling and Force-Position Controller Design of Rehabilitation Robot for Human Arm Movements ", Eng. \& Tech. Journal, Vol. 32, Part (A), No. 8, 2014.

[11] M. Y. Hassan and S. S. Ghintab, "Ant Colony Optimization Based Force- Position Control for Human Lower Limb Rehabilitation Robot", Al-Khwarizmi Engineering Journal, Vol. 12, No. 1, pp. 6172, 2016.

[12] J. Kaushal and S. Ganguli, "Comparative Performance Study of ACO \& ABC Optimization based PID Controller Tuning for Speed Control of DC Motor", M. Sc. Thesis, Thapar University, Patiala, June, 2012.

[13] M. Dorigo and Th. Stutzle, Ant Colony Optimization, Bradford Book, MIT Press, Cambridge, Massachusetts, London, England, 2004.

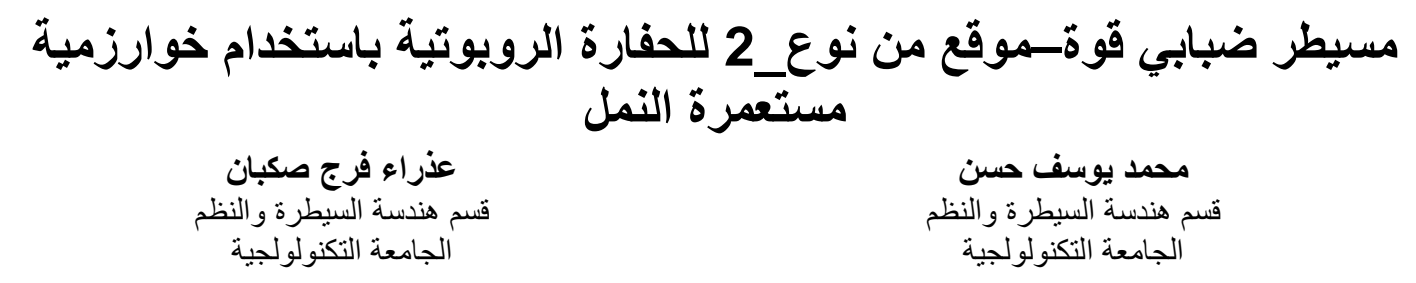

\footnotetext{
الخـــــاصة

يقدم هذا البحث تصميم المسيطر المضبب من نوع -2 للسيطرة على موضع دلو الحفارة الروبوتية خلال عمليات الحفر. للحصدول

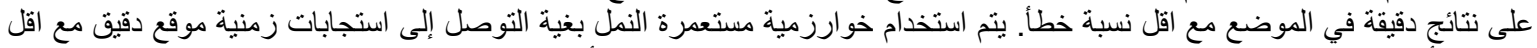

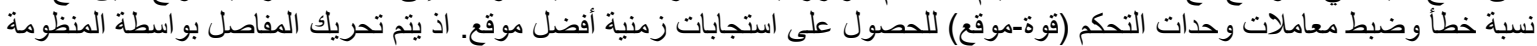

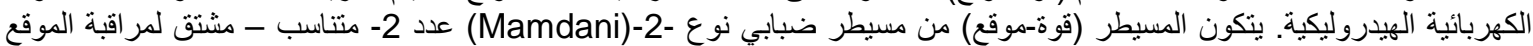

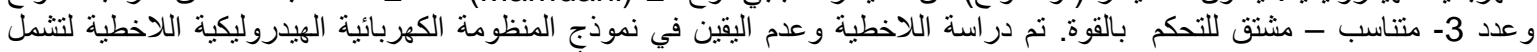

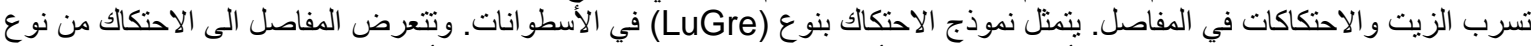
(stribeck-viscous -Coulomb) حصول تحسينات لوحدات التحكم في الموقع بالمقارنة مع وحدات تحكم ضبطها يدوياً داخل النطاق بنسبة (19-23\%)
} 